\title{
Experimental Evaluation of a Model-based Assistance-As-Needed Paradigm using an Assistive Robot
}

\author{
Marc G. Carmichael and Dikai Liu
}

\begin{abstract}
In robotic rehabilitation a promising paradigm is assistance-as-needed. This is because it promotes patient active participation which is essential for neuro-rehabilitation. A model-based assistance-as-needed paradigm has been developed which utilizes a musculoskeletal model representing the subject to calculate their assistance needs. In this paper we experimentally evaluate this model-based paradigm to control an assistive robot and provide a subject with assistance-as-needed at the muscular level. A subject with impairments defined in specific muscle groups performs a number of upper limb tasks, whilst receiving assistance from a robotic exoskeleton. The paradigm is evaluated on its ability to provide assistance only as the subject needs, depending on the tasks being performed and the impairments defined. Results show that the modelbased assistance-as-needed paradigm was relatively successful in providing assistance when it was needed.
\end{abstract}

\section{INTRODUCTION}

New robotic rehabilitation paradigms continue to be developed with the aim of improving patient recovery [1]. The most common paradigms are assistive, with the robot aiding patients as they perform movements as part of therapy. How the robot provides assistance can have a large effect on the patient's recovery. To facilitate motor-neuron recovery following disorders such as stroke it is essential that patients actively participate [2]. Hence paradigms such as assistanceas-needed have shown promise for robotic therapy as they inherently promote patient participation by limiting assistance to the minimum the patient requires. However, identifying their true assistance needs is difficult as physiology varies from person to person, and is exacerbated in rehabilitation due to the patient's impairment. A common solution is to provide performance-based assistance which has shown promising results [3]-[5].

In previous work we developed a model-based paradigm to estimate the assistance needs of a human operator [6]. This paradigm incorporates a musculoskeletal model to calculate the operator's strength [7] which is compared against the requirements of the task being performed [8] to gauge their assistance needs, and subsequently used to govern the assistance provided by a robot. Model-based methods for providing assistance have several benefits. The patient's assistance needs can be gauged from the model without them first performing the task, unlike performance-based methods which require observations of each task before their assistance needs can be inferred from their performance. Additionally, incorporating a musculoskeletal model allows

M. G. Carmichael and D. Liu are with the Centre for Autonomous Systems (CAS), Faculty of Engineering and IT (FEIT), University of Technology, Sydney (UTS), NSW 2007, Australia. Email: marc.carmichael@uts.edu.au, dikai.liu@uts.edu.au analysis relating to the subject's physiology. For example, the model may be used to identify impairment, or to plan optimized therapies [9]-[11]. Although there are many benefits, the efficacy of the model-based paradigm to provide assistance-as-needed has yet to be determined.

In this paper we experimentally evaluate the model-based assistance-as-needed paradigm on its ability to estimate the assistance needs of a subject based on impairments at the muscular level, and to provide assistance accordingly. A healthy subject performs a number of physical tasks utilizing their upper limb. Although the subject is healthy, for the purpose of evaluation they are assigned virtual impairments in their upper limb muscles. The subject's assistance need in each task is calculated using the musculoskeletal model, taking into account the defined impairments. The result is used to govern the assistance provided by a robotic exoskeleton which aids the subject as they perform the tasks. Ideally, the assistance provided should be greater in tasks requiring the use of impaired muscles. Alternatively the assistance should be minimal when tasks are performed which do not require impaired muscles, since the remaining non-impaired muscles are capable of performing it. It is on this basis that the modelbased assistance paradigm is evaluated.

\section{EXPERimental EVAluation of Musculoskeletal MOdEL-BASED AsSistance}

\section{A. Physical tasks utilizing the upper limb}

The experiment involves a healthy subject performing a number of tasks utilizing their upper limb. Each task requires the subject to hold their arm in a specified position whilst statically opposing an external force applied to the hand for approximately two seconds. This external force is applied in one of six orthogonal directions, with each direction constituting a unique task named $T_{1}$ to $T_{6}$, as shown in Fig. 1. The external load has a magnitude of $5 \mathrm{~kg}(49 N)$ as this produces a measurable change in the subject's muscle activity, whilst not being so large as to physically strain them. During each task the exoskeleton assists the subject by supporting a portion of the external load. The amount of assistance provided is calculated using the model-based paradigm, and depends on the task being performed and the muscles which are defined as impaired.

\section{B. Robotic exoskeleton platform}

The specially developed robotic exoskeleton shown in Fig. 2 is used for the experiment. It assists the subject's upper limb with three active degrees of freedom (two shoulder, one elbow) acting as a mechanical interface between them and 

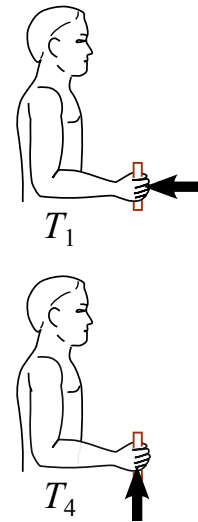
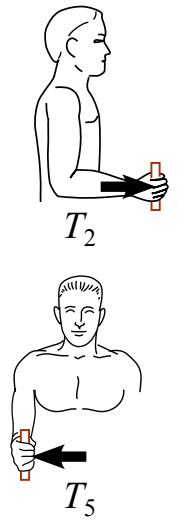
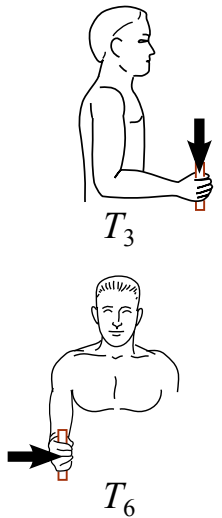

Fig. 1: The six tasks performed in the experiment. All have the upper limb in the same position. Black arrows indicate the direction of the $5 \mathrm{~kg}$ external force applied at the hand.

the task. It supports a portion of the external force applied at their hand, transmitting it to the ground. The exoskeleton operates under an admittance control scheme. The force between the robot and subject is combined with the external force into a net force at the robot's end effector, which is used to generate a velocity response acted out by a PD controller at the joint level. Hence the robot is actively compliant, with its end effector moving in response to both the task's external force and the force applied at the hand by the subject. The interaction force between the subject and robot is measured with a 6-axis force sensor (ATI nano25) fitted in the handle the subject holds. No other physical interaction between the robot and subject occurs apart from at the hand. The task external force is not physically applied to the robot, instead a virtual force is used for convenience. The virtual force is defined in software and fed through the admittance control as if it were a measured external load. The robot acts as if a real load is present, requiring the subject to provide force at the handle to oppose it. Experiments validating the use of virtual external forces showed results were consistent with those obtained using physical external loads.

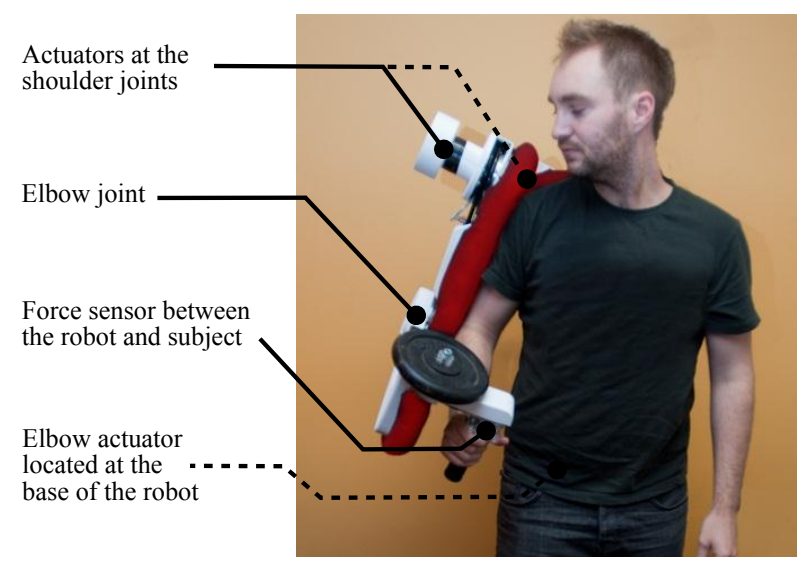

Fig. 2: The exoskeleton used to evaluate the model-based paradigm. Dotted labels indicate obscured components.
The assistance provided by the exoskeleton is controlled by scaling down the task's external force before it is fed into the admittance control using the gain $1-A$, where $0 \leq A \leq 1$. When $A=0$ the external force is not scaled down at all, hence to statically oppose it requires the subject to provide an opposing force of equal magnitude with their hand to achieve zero net force at the end effector. As $A$ is increased, the effort required to oppose the external force decreases, until $A=1$ at which point the external force is scaled down to zero and no effort from the subject is required.

\section{Muscle impairment profiles}

During the experiment the subject is assigned different impairment profiles. Eight profiles (represented as $I_{1}$ to $I_{8}$ ) are created which define physical impairment localized in specific upper limb muscle groups. Impairment is simulated by limiting the active force production in corresponding muscles of the musculoskeletal model to $1 \%$ of their typical capacity when the subject's strength is calculated. Table I lists the muscles impaired in each profile, and the names of the corresponding muscles in the musculoskeletal model used to calculate subject strength [12]. Profile $I_{0}$ represents the subject with no impairment.

TABLE I: Impairment profiles used during the experiment.

\begin{tabular}{|c|c|c|}
\hline $\begin{array}{c}\text { Impairment } \\
\text { profile }\end{array}$ & $\begin{array}{c}\text { Impaired upper } \\
\text { limb muscles }\end{array}$ & $\begin{array}{c}\text { Corresponding muscle names } \\
\text { in the musculoskeletal model }\end{array}$ \\
\hline$I_{0}$ & None & - \\
\hline$I_{1}$ & Biceps & BIClong, BICshort \\
\hline$I_{2}$ & Triceps & TRIlong, TRIlat, TRImed \\
\hline$I_{3}$ & Anterior deltoid & DELT1 \\
\hline$I_{4}$ & Lateral deltoid & DELT2 \\
\hline$I_{5}$ & Posterior deltoid & DELT3 \\
\hline$I_{6}$ & Pectoralis major & PECM1, PECM2, PECM3 \\
\hline$I_{7}$ & Infraspinatus & INFSP \\
\hline$I_{8}$ & Latissimus dorsi & LAT1, LAT2,LAT3 \\
\hline
\end{tabular}

\section{Assistance calculation using the musculoskeletal model}

The subject's assistance need depends on how capable they are of performing each task. It also depends on any impairment they possess, since this affects their capability. Each of the tasks requires strength at the subject's hand for them to be performed. This strength is calculated using an optimization model which makes use of a musculoskeletal model representing the subject's upper limb. For detailed information about the model and how strength is calculated readers are directed to the following references [6]-[8].

A publicly available upper limb musculoskeletal model is used [12] with no adjustments made to fit the model to the subject. Strength is calculated by positioning the upper limb model corresponding to that shown in Fig. 1. The optimization then calculates the maximum magnitude of force the model can oppose at the hand, ensuring that physiological constraints are met. Strength is calculated in the direction corresponding to the external force direction of the task being performed. This calculation is repeated for each of the six tasks $\left(T_{1}\right.$ to $\left.T_{6}\right)$ with each of the nine impairment profiles applied $\left(I_{0}\right.$ to $\left.I_{8}\right)$. The calculated 
strength results for all tasks and impairment profiles are shown in Table II.

TABLE II: Subject's strength capability calculated for each task, and for each impairment profile. Units are in Newtons.

\begin{tabular}{|c||c|c|c|c|c|c|c|c|c|}
\cline { 2 - 11 } \multicolumn{1}{c|}{} & \multicolumn{10}{c|}{ Impairment profile } \\
\hline Task & $I_{0}$ & $I_{1}$ & $I_{2}$ & $I_{3}$ & $I_{4}$ & $I_{5}$ & $I_{6}$ & $I_{7}$ & $I_{8}$ \\
\hline \hline$T_{1}$ & 355 & 290 & 320 & 178 & 270 & 355 & 235 & 275 & 355 \\
\hline$T_{2}$ & 280 & 280 & 214 & 280 & 280 & 238 & 280 & 213 & 209 \\
\hline$T_{3}$ & 247 & 134 & 247 & 147 & 210 & 247 & 190 & 201 & 247 \\
\hline$T_{4}$ & 99 & 99 & 20 & 99 & 99 & 99 & 99 & 99 & 99 \\
\hline$T_{5}$ & 124 & 115 & 117 & 103 & 123 & 124 & 90 & 124 & 102 \\
\hline$T_{6}$ & 100 & 100 & 99 & 99 & 87 & 97 & 99 & 26 & 100 \\
\hline
\end{tabular}

The $A$ parameter which controls the assistance the exoskeleton provides is based on how much each impairment affects the subject's strength. It is calculated as the loss of strength at the hand for the task being performed due to the impairment profile applied, normalized by the strength calculated with no impairment (i.e. $I_{0}$ ). We represent the strength calculated for the $i$-th task with the $j$-th impairment profile applied as $S_{P}^{[i, j]}$. Likewise we represent parameter $A$ calculated for the $i$-th task with the $j$-th impairment profile applied as $A^{[i, j]}$, and is calculated using Eqn (1). The term $S_{P}^{[i, 0]}$ is the calculated strength for the $i$-th task with no impairment applied.

$$
A^{[i, j]}=\frac{S_{P}^{[i, 0]}-S_{P}^{[i, j]}}{S_{P}^{[i, 0]}}
$$

\section{E. Experimental procedure and evaluation}

The subject performs each of the six tasks with each of the nine impairment profiles applied. During each task the exoskeleton provides assistance by supporting a portion of the external force. Consultation was made with the UTS Human Research Ethics Committee (HREC).

The amount of assistance is governed by parameter $A$, which is calculated as previously described. During each task the subject's muscle activity is measured using electromyography (EMG). Eight upper limb muscle groups are measured, corresponding to the same impaired muscles in impairment profiles $I_{1}$ to $I_{8}$ (see Table I). EMG is measured using the Delsys Bagnoli system sampled at $10 \mathrm{kHz}$, notch filtered to remove any mains power noise, high-pass filtered, rectified, and low-pass filtered to produce a linear envelope. This is then normalized using prior measurements obtained during maximum voluntary contractions in each muscle to produce a normalized result ranging from 0 to 1 indicating the utilization of each muscle.

Evaluation of the model-based paradigm is made by correlating when the assistance is needed, to when the assistance is provided by the exoskeleton. The exact relationship between impairments at the muscular level and the resulting assistance need when performing tasks at the hand is not known. However it is logical that the more a muscle is utilized during a task, the more that task is sensitive to impairments in that muscle. Using this rationale, the EMG measured for each task when no assistance is provided (i.e. $A=0$ ) is used to infer how much assistance should be received if that same muscle was impaired. A task in which a muscle is utilized a lot should receive greater assistance if that same muscle was impaired. Alternatively, for a task in which an impaired muscle is not utilized, then less assistance should be provided since the subject should still be able to perform that task using the remaining non-impaired muscles. We represent the EMG of the $m$-th muscle, measured during the $i$-th task with no assistance provided as $E^{[i, m]}$. The correlation between the assistance provided and the inferred assistance needed is then calculated on a muscle per muscle basis using Eqn (2) where $j=m$ is the muscle it is calculated with respect to.

$$
\rho=\operatorname{corr}\left(\left\{A^{[1, j]}, \ldots, A^{[6, j]}\right\},\left\{E^{[1, m]}, \ldots, E^{[6, m]}\right\}\right)
$$

\section{RESUlts AND Discussion}

In Fig. 3 the assistance provided $(A)$, and the measured EMG $(E)$ from which we infer the assistance needed, are plotted side by side for all the tasks and each of the eight muscles groups. Since the assistance need is inferred from the measured EMG it is not expected that the magnitudes of these two data sets match, instead the evaluation is made based on their correlation. Hence the two sets are plotted with different vertical scales so as to allow a visual comparison. Also shown are the correlation results calculated with respect to each muscle.

The biceps, triceps, anterior deltoid, pectoralis major, and infraspinatus muscles all produce correlation results greater than 0.5 . Visually comparing the results for these muscles a noticeable similarity between the measured EMG and the assistance provided is observed. The lateral deltoid, posterior deltoid and latissimus dorsi muscles show less similarity, which is reflected by the lower correlation results calculated. However all of the results were above zero, the lowest being 0.14 for the lateral deltoid. Possibly contributing to some muscles achieving poorer results is certain physiological factors not being considered when the subject's strength is calculated. The lateral deltoid, posterior deltoid and latissimus dorsi muscles have been identified as contributing to shoulder stability or instability [13], [14], hence it is speculated that not considering joint stability when estimating upper limb strength may be a factor in why these muscles achieved lower correlation compared to other muscles.

Overall the results suggest the model-based paradigm was reasonably successful in predicting the tasks most affected by particular muscular impairments, and then providing assistance accordingly. We find these results encouraging as no adjustments were made to fit the generic upper limb model to represent the subject, yet positive results were achieved. This encourages further research into the use of model-based paradigms for providing assistance-as-needed. Presently, impairments are defined in specific muscle groups without a basis on individual subjects. Future work is required to 
investigate how results differ with the musculoskeletal model and the impairment profiles made subject specific, based on actual impaired subjects. Also planned is the incorporation of additional physiological factors such as shoulder stability into the strength calculation.

\section{Biceps \\ $(\rho=0.84)$}
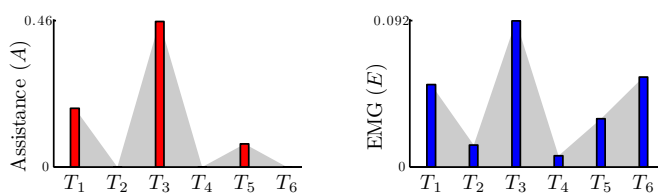

Triceps

$(\rho=0.72)$
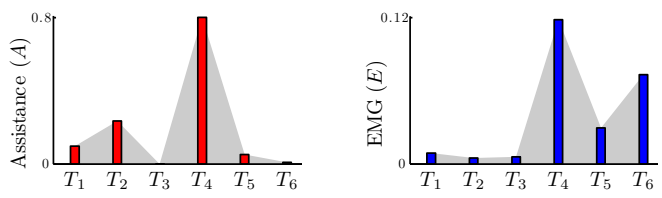

Anterior

Deltoid

$(\rho=0.92)$
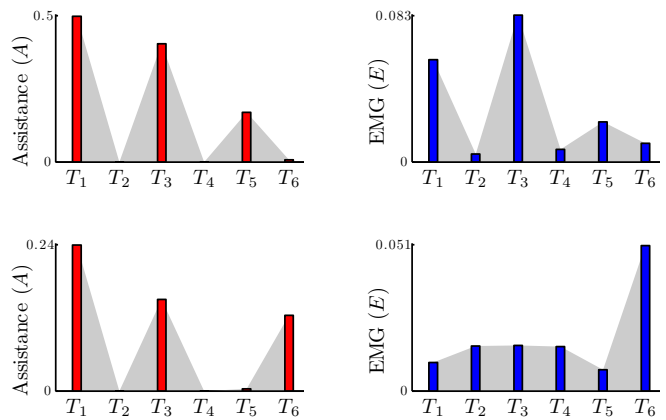

Lateral

Deltoid

$(\rho=0.14)$

Posterior

$(\rho=0.30)$
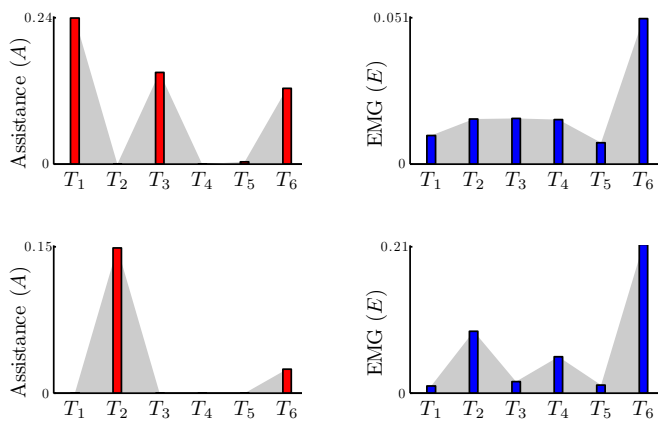

Pectoralis
Major
$(\rho=0.52)$
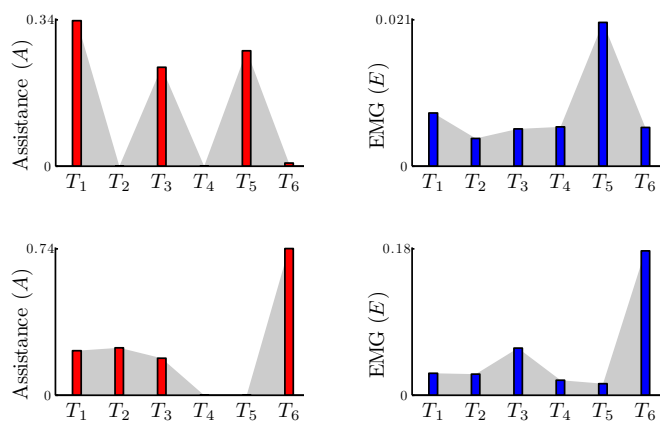

Infraspinatus

$(\rho=0.94)$

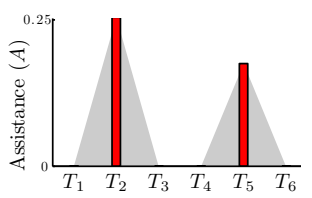

\section{CONCLUSION}

Experimental evaluation of a model-based paradigm for providing robotic assistance to specific muscles was performed. The paradigm was implemented on a robotic exoskeleton to assist a subject as they performed a number of physical upper limb tasks. The subject was assigned impairments localized to specific upper limb muscle groups. The paradigm was evaluated on its ability to provide assistance as needed, i.e. provide greater assistance when tasks which require impaired muscles were performed, and provide minimal assistance for tasks that do not utilize impaired muscles. Results indicate the paradigm was reasonably effective in estimating the subject's assistance needs and provided assistance accordingly.

\section{REFERENCES}

[1] L. Marchal-Crespo and D. J. Reinkensmeyer, "Review of control strategies for robotic movement training after neurologic injury," Journal of NeuroEngineering and Rehabilitation, vol. 6, no. 1, p. 20, 2009.

[2] N. Hogan, H. I. Krebs, B. Rohrer, J. J. Palazzolo, L. Dipietro, S. E. Fasoli, J. Stein, R. Hughes, W. R. Frontera, D. Lynch, and B. T. Volpe, "Motions or muscles? Some behavioral factors underlying robotic assistance of motor recovery." Journal of rehabilitation research and development, vol. 43, no. 5, pp. 605-618, 2006.

[3] H. Krebs, J. Palazzolo, L. Dipietro, M. Ferraro, J. Krol, K. Rannekleiv, B. Volpe, and N. Hogan, "Rehabilitation robotics: Performance-based progressive robot-assisted therapy," Autonomous Robots, vol. 15, pp. 7-20, 2003.

[4] J. Emken, J. Bobrow, and D. Reinkensmeyer, "Robotic movement training as an optimization problem: designing a controller that assists only as needed," in 9th International Conference on Rehabilitation Robotics (ICORR), 2005, June 2005, pp. 307 - 312.

[5] E. Wolbrecht, V. Chan, V. Le, S. Cramer, D. Reinkensmeyer, and J. Bobrow, "Real-time computer modeling of weakness following stroke optimizes robotic assistance for movement therapy," in Neural Engineering, 2007. CNE '07. 3rd International IEEE/EMBS Conference on, May 2007, pp. $152-158$.

[6] M. G. Carmichael and D. Liu, "Estimating physical assistance need using a musculoskeletal model," IEEE Transactions on Biomedical Engineering, 2013, (in press).

[7] — - "Towards using musculoskeletal models for intelligent control of physically assistive robots," in Engineering in Medicine and Biology Society,EMBC, 2011 Annual International Conference of the IEEE, Sept 2011, pp. $8162-8165$.

[8] — "A task description model for robotic rehabilitation," in Engineering in Medicine and Biology Society,EMBC, 2012 Annual International Conference of the IEEE, 2012.

[9] J. Ueda, D. Ming, V. Krishnamoorthy, M. Shinohara, and T. Ogasawara, "Individual muscle control using an exoskeleton robot for muscle function testing," Neural Systems and Rehabilitation Engineering, IEEE Transactions on, vol. 18, no. 4, pp. 339 -350, Aug. 2010.

[10] B. J. Fregly, M. L. Boninger, and D. J. Reinkensmeyer, "Personalized neuromusculoskeletal modeling to improve treatment of mobility impairments: a perspective from european research sites." J Neuroeng Rehabil, vol. 9, p. 18, 2012.

[11] Y. Pei, Y. Kim, G. Obinata, E. Genda, and D. Stefanov, "Robot-aided rehabilitation task design for inner shoulder muscles," in Engineering in Medicine and Biology Society (EMBC), 2012 Annual International Conference of the IEEE, Sep 2012, pp. $3922-3925$.

[12] K. R. S. Holzbaur, W. M. Murray, and S. L. Delp, "A model of the upper extremity for simulating musculoskeletal surgery and analyzing neuromuscular control," Annals of Biomedical Engineering, vol. 33, no. 6, pp. 829-840, June 2005.

[13] D. C. Ackland and M. G. Pandy, "Lines of action and stabilizing potential of the shoulder musculature." J Anat, vol. 215, no. 2, pp. 184-197, Aug 2009.

[14] F. Steenbrink, J. de Groot, H. Veeger, F. van der Helm, and P. Rozing, "Glenohumeral stability in simulated rotator cuff tears," Journal of Biomechanics, vol. 42, no. 11, pp. 1740 - 1745, 2009. 